\title{
How many spinal cord injury patients can catheterize their own bladder? The epidemiology of upper extremity function as it affects bladder management
}

\author{
DV Zlatev ${ }^{1}, \mathrm{~K}$ Shem ${ }^{2}$ and CS Elliott ${ }^{1,3}$
}

Study design: Retrospective analysis.

Objectives: In patients with spinal cord injury, limitations in upper extremity (UE) motor function are cited as a reason for the lack of adherence to clean intermittent catheterization (CIC). By examining the UE function in spinal cord injury (SCI) patients, we aim to provide insight into why $\mathrm{CIC}$ 'dropout' occurs and determine a more appropriate target percentage for $\mathrm{CIC}$ in this patient population. Setting: United States centers participating in National Spinal Cord Injury Database (NSCID).

Methods: We assessed discharge data from the 2006 to 2012 NSCID. Neurologic motor scores for C5 to C8 (involved in UE movement) were transformed into a binary variable consisting of the ability ('strong') or the inability ('weak') to achieve active motion against resistance. We generated an algorithm based on expert opinion and published literature to categorize a person's ability to perform $\mathrm{CIC}$ by the UE function alone.

Results: Of the 4481 patients evaluated, $77.3 \%$ were unable to volitionally void. Of this subset, $58.8 \%$ were categorized as able to catheterize, $12.9 \%$ as possibly able, $4.3 \%$ as only able with surgical assistance and $23.3 \%$ as unable. Among patients discharged with an indwelling catheter, $33.4 \%$ had adequate UE function for $\mathrm{CIC}$. Among patients performing $\mathrm{CIC}$ at discharge, $14.1 \%$ had inadequate UE function for self-catheterization.

Conclusions: CIC dropout may occur at least, in part, because of inadequate UE motor function. In a 'best-case' scenario, 76\% of patients with $\mathrm{SCl}$ who cannot volitionally void could potentially perform $\mathrm{CIC}$ given appropriate assistance.

Spinal Cord (2016) 54, 287-291; doi:10.1038/sc.2015.169; published online 19 January 2016

\section{INTRODUCTION}

Roughly 270000 persons in the United States have sustained a spinal cord injury (SCI), with an estimated 12000 new cases occurring annually. ${ }^{1}$ The majority of patients with SCI have bladder dysfunction because of their injury, with over $75 \%$ unable to volitionally void. ${ }^{2}$ As Lapides et al. ${ }^{3}$ published their seminal work in 1972, clean intermittent catheterization (CIC) has been championed as the 'gold standard' in what patients and physicians should strive to achieve for neurogenic bladder management. ${ }^{3}$ Compared with indwelling catheters, condom catheters and reflex voiding, there are significantly fewer complications in persons performing CIC. ${ }^{4-6}$ However, many patients with SCI have difficulty in performing CIC. This includes patients with (a) limited upper extremity (UE) motor function, (b) anatomic limitations that make accessing the urethra difficult (e.g., females or obese persons) and (c) limited functional bladder capacity (due to detrusor overactivity or poor bladder compliance). Thus, in many instances, CIC may not be feasible from a functional or a social standpoint.

To regain bladder independence, many patients frequently opt for indwelling urethral or suprapubic urinary catheters. Although nearly $60 \%$ of patients who are unable to volitionally void after SCI are performing $\mathrm{CIC}$ at the time of discharge from rehabilitation, only half of this subset continue to do so at 5-year follow-up. ${ }^{2}$ Although urologic surgical intervention can help patients with incontinence or certain anatomic problems, UE function is in most instances irreparable.

The purpose of this study is to provide an in-depth look at the UE motor function in the SCI population at the time of discharge from rehabilitation, with additional attention to other anatomic factors such as gender and body mass index (BMI) that might affect selfcatheterization. From this information, we hope to provide insight into why CIC 'dropout' occurs and to more accurately determine a 'true' target percentage for CIC in the SCI population.

\section{PATIENTS AND METHODS}

We assessed Form I data for the years 2006 to 2012 from the National Spinal Cord Injury Database (NSCID), a large database that includes medical and demographic data on patients with SCI in designated Model Spinal Cord Injury Systems facilities in the United States. ${ }^{7}$ Form I is administered during a patient's initial rehabilitation process, before discharge from an in-patient rehabilitation service. As part of the evaluation, a complete neurologic motor exam is administered at the time of discharge, including a separate assessment of the left and right side from $\mathrm{C} 5$ to $\mathrm{C} 8$. The motor scores for each side and level are graded individually from 0 to $5(0=$ total paralysis; $1=$ palpable or visible contraction; 2 = active movement, full range of motion with gravity eliminated; $3=$ active movement, full range of motion against gravity; $4=$ active movement,

\footnotetext{
${ }^{1}$ Department of Urology, Stanford University School of Medicine, Stanford, CA, USA; ${ }^{2}$ Department of Physical Medicine and Rehabilitation, Santa Clara Valley Medical Center, San Jose, CA, USA and ${ }^{3}$ Department of Urology, Santa Clara Valley Medical Center, San Jose, CA, USA

Correspondence: Dr DV Zlatev, Department Urology, Stanford University School of Medicine, 300 Pasteur Drive, Grant S-287, Stanford, CA 94305-5118, USA.

E-mail: dzlatev@stanford.edu

Received 25 April 2015; revised 22 August 2015; accepted 1 September 2015; published online 19 January 2016
} 
full range of motion against moderate resistance; $5=$ active movement, full range of motion against full resistance). Form I additionally includes patient demographics, patient health information and bladder management method at discharge.

We transformed the neurologic motor scores at levels C5 to C8 (involved in the control of movement of the UEs) into a binary variable, consisting of the ability or the inability to achieve active motion against moderate or full resistance and reclassified them as 'strong' (manual muscle test grade 4 or 5) and 'weak' (manual muscle test grade $0,1,2$ or 3), respectively. Using these scores and expert opinion, we characterized a person's ability to self-catheterize based only on UE motor strength. This was categorized as follows: (a) able to catheterize, (b) possibly able to catheterize, (c) able to catheterize only with reconstructive surgical intervention (e.g., catheterizable stoma construction or tendon transfer surgery) and (d) unable to catheterize even with reconstructive surgical intervention. Our algorithm for classifying the ability to perform CIC is shown in Figure 1.

Patients were excluded from the study if a motor examination was not recorded before discharge or if a patient was younger than 13 years of age. Cross-sectional associations among demographic characteristics, health characteristics and bladder management methods were tested using the $\chi^{2}$ test. All data manipulation and statistics were performed using Stata version 12.1 (StataCorp, College Station, TX, USA).

\section{RESULTS}

Form I data were available for 5018 patients in the NSCID for the years 2006 to 2012. After exclusions, a total of 4481 patients were evaluated, including $1016(22.7 \%)$ patients who were able to volitionally void and $3465(77.3 \%)$ patients who were unable to volitionally void. Demographic characteristics and overall SCI levels were similar between all patients and the subgroup of patients who were unable to volitionally void at discharge (Table 1). A significant difference in bladder management at the time of discharge was noted on the basis of American Spinal Injury Association Impairment Scale. Greater than $85 \%$ of patients able to void volitionally at discharge were classified as American Spinal Injury Association Impairment Scale D, whereas $<20 \%$ of patients who were discharged with a non-volitional bladder management were classified as American Spinal Injury Association Impairment Scale D $(P<0.001)$.

The bladder management of patients unable to volitionally void at the time of discharge from rehabilitation is shown in Table 2. The percentage of individuals whose bladder was managed with an indwelling catheter was higher in women compared with that in men $(42.9 \%$ vs $29.3 \%, P<0.001)$, whereas the percentage of individuals whose bladder was managed with CIC was lower in women compared with that in men $(49.5 \%$ vs $61.5 \%, P<0.001)$. For patients who were unable to volitionally void, CIC (59.2\%) was more commonly used compared with indwelling catheterization $(31.9 \%)$ or condom catheterization $(2.5 \%)$.

On the basis of our assessment of an individual's UE function, patients who were discharged performing CIC were more likely to have adequate UE function to self-catheterize compared with those discharged with an indwelling catheter $(72.0 \%$ vs $33.4 \%, P<0.001)$ (Table 3). It is notable, however, that of patients discharged with an indwelling catheter more than one-third had adequate UE function for CIC, with an additional $17.1 \%$ possibly able to perform CIC and another $6.4 \%$ potentially able to catheterize with surgical intervention. Thus, a total of $56.9 \%$ of patients discharged with an indwelling catheter had UE function that did not preclude bladder management with CIC. Conversely, in the group performing CIC at discharge, $14.1 \%$ had inadequate UE function for self-catheterization and another $3 \%$ would be unable to self-catheterize without surgical intervention.

Among all patients who could not volitionally void at discharge from rehabilitation, $58.8 \%$ were able to catheterize by our UE function criteria alone, $12.9 \%$ were possibly able to catheterize and $4.3 \%$ would be able to catheterize with surgical assistance. Only $23.3 \%$ of these

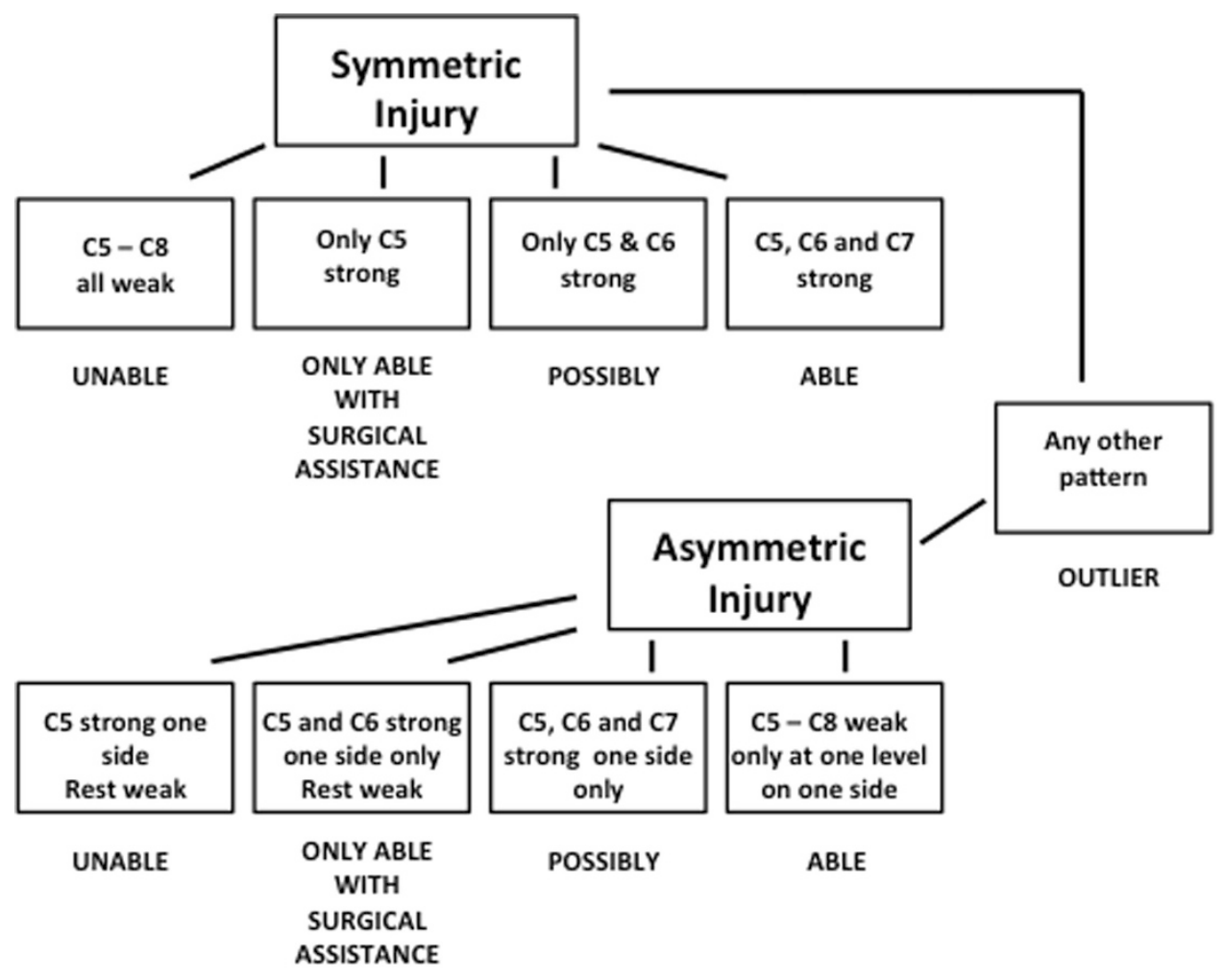

Figure 1 Algorithm for the ability to self-catheterize based on UE motor strength scores. 
Table 1 Demographics and $\mathrm{SCl}$ characteristics

\begin{tabular}{|c|c|c|}
\hline Characteristic & $\begin{array}{l}\text { All bladder manage- } \\
\text { ment }(\mathrm{n}=4481)\end{array}$ & $\begin{array}{l}\text { Non-volitional bladder } \\
\text { management }(\mathrm{n}=3465)\end{array}$ \\
\hline \multicolumn{3}{|l|}{ Age (years) } \\
\hline Median & 41 & 38 \\
\hline Range & 13-95 & 13-95 \\
\hline \multicolumn{3}{|l|}{ Gender, no. (\%) } \\
\hline Male & 3575 (79.8) & $2800(80.8)$ \\
\hline Female & $906(20.2)$ & $665(19.2)$ \\
\hline \multicolumn{3}{|l|}{$B M{ }^{a}$, no. (\%) } \\
\hline $\begin{array}{l}\text { Underweight or } \\
\text { normal }\end{array}$ & $2196(49.0)$ & 1717 (49.6) \\
\hline Overweight & $1285(28.7)$ & $965(27.8)$ \\
\hline Obese & $586(13.1)$ & 458 (13.2) \\
\hline $\begin{array}{l}\text { Morbidly or super } \\
\text { obese }\end{array}$ & $414(9.2)$ & $325(9.4)$ \\
\hline \multicolumn{3}{|l|}{ Etiology, no. (\%) } \\
\hline $\begin{array}{l}\text { GSW, penetrating } \\
\text { injury }\end{array}$ & 605 (13.5) & 517 (14.9) \\
\hline Vehicular & $1813(40.5)$ & 1431 (41.3) \\
\hline Sports & $380(8.5)$ & $300(8.7)$ \\
\hline Fall & $1275(28.5)$ & $920(26.6)$ \\
\hline Medical & $229(5.1)$ & $166(4.8)$ \\
\hline Other & $179(4.0)$ & $131(3.8)$ \\
\hline \multicolumn{3}{|c|}{ Marital status, no. (\%) } \\
\hline Single & $2054(45.8)$ & $1651(47.6)$ \\
\hline Married & $1663(37.1)$ & 1257 (36.3) \\
\hline Divorced & 477 (10.6) & 343 (9.9) \\
\hline Other & $287(6.4)$ & $214(6.2)$ \\
\hline \multicolumn{3}{|l|}{ Time from injury, days } \\
\hline Median & 66 & 73 \\
\hline Range & $7-580$ & $8-580$ \\
\hline \multicolumn{3}{|c|}{ Neurologic level of SCI, no. (\%) } \\
\hline C1 & $85(1.9)$ & $64(1.8)$ \\
\hline $\mathrm{C} 2$ & $189(4.2)$ & $131(3.8)$ \\
\hline C3 & $264(5.9)$ & $186(5.4)$ \\
\hline C4 & $793(17.7)$ & $636(18.4)$ \\
\hline C5 & $623(13.9)$ & $420(12.1)$ \\
\hline C6 & $307(6.9)$ & $229(6.6)$ \\
\hline C7 & $163(3.6)$ & $117(3.4)$ \\
\hline $\mathrm{C} 8$ & $76(1.7)$ & $57(1.6)$ \\
\hline $\mathrm{T} 1$ & $85(1.9)$ & $62(1.8)$ \\
\hline Other & $1896(42.3)$ & $1563(45.1)$ \\
\hline \multicolumn{3}{|c|}{ ASIA impairment scaleb, no. (\%) } \\
\hline A & $1589(35.5)$ & $1579(45.6)$ \\
\hline B & $572(12.8)$ & $561(16.2)$ \\
\hline C & $702(15.7)$ & $597(17.2)$ \\
\hline $\mathrm{D}$ & $1539(34.3)$ & $675(19.5)$ \\
\hline $\mathrm{E}$ & $18(0.4)$ & $3(0.1)$ \\
\hline Not done & $61(1.4)$ & $50(1.4)$ \\
\hline
\end{tabular}

Abbreviations: ASIA, American Spinal Injury Association; BMI, body mass index; GSW, gunshot wound; $\mathrm{SCl}$, spinal cord injury.

anderweight individuals classified as BMI $<18.5 \mathrm{~kg} \mathrm{~m}^{-2}$; normal weight individuals classified as BMI 18.5-25 $\mathrm{kg} \mathrm{m}^{-2}$; overweight individuals classified as BMI 25-30 $\mathrm{kg} \mathrm{m}^{-2}$; obese individuals classified as BMI $30-35 \mathrm{~kg} \mathrm{~m}^{-2}$; morbidly obese individuals classified as BMI $35-45 \mathrm{~kg} \mathrm{~m}^{-2}$; super obese individuals classified as BMI $>45 \mathrm{~kg} \mathrm{~m}^{-2}$.

${ }^{\mathrm{b}} \mathrm{A}=$ complete injury, no sensory or motor function preserved in sacral segments $\mathrm{S} 4-\mathrm{S} 5$; $\mathrm{B}=$ incomplete injury, sensory but not motor function preserved below neurological level and sacral segments S4-S5; C = incomplete injury, motor function preserved below neurological level and more than half of key muscles below neurological level have muscle grade $<3$;

$\mathrm{D}=$ incomplete injury, motor function preserved below neurological level and at least half of key muscles below neurological level have muscle grade $\geqslant 3 ; E=$ normal sensory and motor functions.

patients would be completely unable to independently perform CIC on the basis of UE function criteria.

BMI and gender were also evaluated as potential barriers to CIC. Among SCI patients who could not volitionally void, $22.6 \%$ were classified as obese, morbidly obese or super obese. Female patients were more likely to be obese (BMI $30 \mathrm{~kg} \mathrm{~m}^{-2}$ or more) compared
Table 2 Bladder management in non-volitional voiders at discharge from rehabilitation

\begin{tabular}{lccr}
\hline Bladder management & Male, no. (\%) & Female, no. (\%) & All, no. (\%) \\
\hline None, other & $164(5.9)$ & $49(7.4)$ & $213(6.1)$ \\
Indwelling catheter & $820(29.3)$ & $285(42.9)$ & $1105(31.9)$ \\
Condom catheter & $85(3.0)$ & $0(0.0)$ & $85(2.5)$ \\
ClC & $1723(61.5)$ & $329(49.5)$ & $2052(59.2)$ \\
Urinary diversion & $8(0.3)$ & $2(0.3)$ & $10(0.3)$ \\
Total & 2800 & 665 & 3465 \\
\hline
\end{tabular}

Abbreviation: CIC, clean intermittent catheterization.

with male patients $(30.5 \%$ vs $20.7 \%, P<0.001)$ (Table 4$)$. BMI appeared to have a significant impact on bladder management at discharge. In women with BMI $>25 \mathrm{~kg} \mathrm{~m}^{-2}$ (overweight, obese or morbidly/super obese), the rate of CIC bladder management decreased significantly, and the rate of IC bladder management increased significantly $(P<0.001$ for both). In men, increases in BMI had no significant association with the choice of bladder management at hospital discharge. In addition, there was no statistical difference in the UE function between males and females or among different classifications of BMI ( $P>0.05$ for all groups) (data not shown).

In a 'best-case' scenario among SCI patients who could not volitionally void at the time of discharge and based on our algorithm were classified as able, possibly able or only able to catheterize with surgical assistance, $\sim 76 \%$ had the UE function to potentially perform CIC given appropriate patient motivation, an adequate home support system and prosthetic or surgical assistance (i.e. tenodesis aids, tendon transfer, construction of catheterizable stoma). Conversely, in a 'worstcase' scenario assuming all females, all obese or morbidly/super obese males and anyone with non-optimal hand function (possibly able to catheterize or able to catheterize only with surgical assistance) could not catheterize independently, only $\sim 38 \%$ of SCI patients could potentially perform CIC.

\section{DISCUSSION}

Although CIC is widely accepted as the bladder management method with lowest long-term morbidity, the fact that up to $50 \%$ of patients abandon its use within 5 years of follow-up suggests that investigation must be carried out to understand why this occurs. On the basis of previous data, inadequate UE function is a significant barrier to a patient's adopting of $\mathrm{CIC}^{8}$ and might have a prominent role in this 'dropout'. In our study, $58.8 \%$ of SCI patients who are unable to volitionally void possess the UE function adequate enough to independently perform CIC, whereas $23.3 \%$ lack the UE function to self-catheterize. These rates are similar to the actual bladder management at discharge from rehabilitation for these patients (59\% CIC, $32 \%$ indwelling catheter). It is notable, however, that our UE function algorithm proposes that up to $14 \%$ of patients discharged home with CIC for bladder management are likely unable to self-catheterize and another $3 \%$ would require surgical intervention to successfully self-catheterize. This suggests that CIC 'dropout' may be, in part, because of a patient's physical inability to independently perform self-catheterization. These UE function data also suggest that the percentage of patients discharged with an indwelling catheter for bladder management has the potential to be decreased, as $>33 \%$ of these patients have adequate UE function to perform CIC and another $23 \%$ are possibly able or able with surgical assistance.

Although the UE function was similar in male and female SCI patients, the $10 \%$ difference in CIC and IC rates between the groups 
Table 3 Predicted ability to self-catheterize based on upper extremity function ${ }^{\text {a }}$

\begin{tabular}{|c|c|c|c|c|c|}
\hline Ability to self-catheterize & Male, no. (\%) & Female, no. (\%) & IC, no. (\%) & CIC, no. (\%) & All, no. (\%) \\
\hline Able & $1625(58.0)$ & $413(62.1)$ & $369(33.4)$ & 1478 (72.0) & $2038(58.8)$ \\
\hline Possibly able & 357 (12.8) & 89 (13.4) & 189 (17.1) & 207 (10.1) & 446 (12.9) \\
\hline Unable & $677(24.2)$ & $130(19.5)$ & $472(42.7)$ & $290(14.1)$ & $807(23.3)$ \\
\hline Outlier & $22(0.8)$ & $4(0.6)$ & $4(0.4)$ & $16(0.8)$ & $26(0.8)$ \\
\hline Total & 2800 & 665 & 1105 & 2052 & 3465 \\
\hline
\end{tabular}

Abbreviations: CIC, clean intermittent catheterization; IC, indwelling catheter.

a In non-volitional voiders at discharge from rehabilitation stratified by gender and discharge bladder management.

Table 4 Bladder management at discharge from rehabilitation ${ }^{a}$

\begin{tabular}{llrrrr}
\hline BMI & Gender & IC, no. (\%) & CIC, no. (\%) & $\begin{array}{r}\text { CC/other }, \\
\text { no. (\%) }\end{array}$ & All, no. \\
\hline Under/normal & Male & $404(28.8)$ & $863(61.6)$ & $135(9.6)$ & 1402 \\
& Female & $110(34.9)$ & $182(57.8)$ & $23(7.3)$ & 315 \\
& & & & & \\
Overweight & Male & $228(27.9)$ & $513(62.7)$ & $77(9.4)$ & 818 \\
& Female & $69(46.9)$ & $67(45.6)$ & $11(7.5)$ & 147 \\
Obese & Male & $109(30.4)$ & $221(61.7)$ & $28(7.8)$ & 358 \\
& Female & $48(48.0)$ & $43(43.0)$ & $9(9.0)$ & 100 \\
Morbid/super & Male & $79(35.6)$ & $126(56.8)$ & $17(7.7)$ & 222 \\
obese & & & & & \\
& Female & $58(56.3)$ & $37(35.9)$ & $8(7.8)$ & 103
\end{tabular}

Abbreviations: BMI, body mass index; CC, condom catheter; CIC, clean intermittent catheterization; IC, indwelling catheter.

a For patients whose bladder was not managed by volitional voiding, categorized by BMI and gender.

b Other includes bladder management with urinary diversion or patients with neurogenic bladder who do not follow any established program of bladder management.

suggests that anatomic issues also affect the adoption of CIC. Our subanalysis suggests that BMI may further have a role in this difference, as significantly more female SCI patients were classified as obese or morbidly/super obese as compared with males. Although an increase in BMI does not significantly affect CIC adoption in men, women were significantly affected once their BMI exceeded $25 \mathrm{~kg} \mathrm{~m}^{-2}$.

This study was also intended to help quantify a reasonable proportion of SCI patients who could be discharged on CIC. On the basis of the UE function alone, our results suggest that a number approaching $60 \%$ is reasonable. However, with more effective intervention, this number could be increased to $75 \%$. Prior studies have suggested that modalities such as occupational therapy training, handgrip aids, tendon transfer surgery and catheterizable stoma construction are effective ${ }^{9-13}$ but often underutilized. ${ }^{14}$ Importantly, the UE function is not the only barrier patients have to the adoption of CIC. Anatomic considerations (gender, BMI), ${ }^{2,13}$ uncontrolled incontinence ${ }^{15}$ and cognitive impairment following concomitant brain injury ${ }^{16}$ may also affect the adoption and continuance of CIC. Thus, increased attention to these areas is necessary.

Our findings and conclusions can be criticized from the perspective that a standard algorithm for the UE function necessary to perform self-catheterization has not been previously constructed nor tested. Thus, we mainly rely on expert opinion for our categorization scheme. However, the reported CIC rates in a recent small case series compared favorably with our algorithm (C4-unable to do CIC, C5-36.4\% CIC, C6-66.7\% CIC and C7-85.7\% CIC). ${ }^{17}$

The NCSID does not contain data for several characteristics that influence the choice of the bladder management method, including uncontrolled urinary incontinence, patient motivation, adequate home support system and comprehensive brain injury data that could affect one's cognitive ability to independently perform CIC. The NCSID also cannot account for varying institutional policies regarding the engagement and motivation of patients to perform CIC, which might provide alternative reasons for discharge of patients with adequate UE motor function on a bladder management other than CIC. Finally, longitudinal follow-up of patients to assess whether a poor UE function is indeed a risk factor for CIC 'dropout' has not yet been performed.

Despite these limitations, our study is the first to comprehensively evaluate the UE motor function and its relation to bladder management in the SCI population. This study is further strengthened by a large number of patients and includes data from complete and standardized motor strength exams. As the NCSID encompasses $\sim 15 \%$ of the SCI population of the United States, the study sample is also highly representative. ${ }^{1}$

\section{CONCLUSION}

CIC dropout may occur at least, in part, because of inadequate UE motor function. Future follow-up will be needed to further validate this concept. In a 'best-case' scenario, $\sim 76 \%$ of SCI patients who cannot volitionally void could potentially perform CIC given appropriate assistance.

\section{DATA ARCHIVING}

There were no data to deposit.

\section{CONFLICT OF INTEREST}

The authors declare no conflict of interest.

\section{ACKNOWLEDGEMENTS}

This work was supported, in part, by Grant No. H133A110002 from the National Institute on Disability and Rehabilitation Research, Office of Special Education and Rehabilitation Services, United States Department of Education, Washington DC. The authors certify that all applicable institutional and governmental regulations concerning the ethical use of human volunteers were followed during the course of this research.

1 Spinal cord injury facts and figures at a glance. J Spinal Cord Med 2013; 36: 170-171.

2 Cameron AP, Wallner LP, Tate DG, Sarma AV, Rodriguez GM, Clemens JQ. Bladder management after spinal cord injury in the United States 1972 to 2005. J Urol 2010; 184: 213-217. 
3 Lapides J, Diokno AC, Silber SJ, Lowe BS. Clean, intermittent self-catheterization in the treatment of urinary tract disease. J Urol 1972; 107: 458-461.

4 Ord J, Lunn D, Reynard J. Bladder management and risk of bladder stone formation in spinal cord injured patients. J Urol 2003; 170: 1734-1737.

5 Weld KJ, Dmochowski RR. Effect of bladder management on urological complications in spinal cord injured patients. J Urol 2000; 163: 768-772.

6 Bennett CJ, Young MN, Adkins RH, Diaz F. Comparison of bladder management complication outcomes in female spinal cord injury patients. J Urol 1995; 153: 1458-1460.

7 Spinal Cord Injury Information Network. National Spinal Cord Injury Database. Available at: http://www.spinalcord.uab.edu/show.asp?durki $=24480$. Accessed on 26 August 2015.

8 Yilmaz B, Akkoc Y, Alaca R, Erhan B, Gunduz B, Yildiz N et al. Intermittent catheterization in patients with traumatic spinal cord injury: obstacles, worries, level of satisfaction. Spinal cord 2014; 52: 826-830.

9 Adler US, Kirshblum SC. A new assistive device for intermittent self-catheterization in men with tetraplegia. J Spinal Cord Med 2003; 26: 155-158.

10 Bernuz B, Guinet A, Rech C, Hugeron C, Even-Schneider A, Denys P et al. Selfcatheterization acquisition after hand reanimation protocols in C5-C7 tetraplegic patients. Spinal Cord 2011; 49: 313-317.
11 Gregersen H, Lybaek M, Johannesen IL, Leicht P, Nissen UV, Biering-Sorensen F. Satisfaction with upper extremity surgery in individuals with tetraplegia. J Spinal Cord Med 2014; 38: 161-169.

12 Hakenberg OW, Ebermayer J, Manseck A, Wirth MP. Application of the Mitrofanoff principle for intermittent self-catheterization in quadriplegic patients. Urology 2001; 58: $38-42$.

13 Walsh K, Troxel SA, Stone AR. An assessment of the use of a continent catheterizable stoma in female tetraplegics. BJU Int 2004; 94: 595-597.

14 Bryden AM, Wuolle KS, Murray PK, Peckham PH. Perceived outcomes and utilization of upper extremity surgical reconstruction in individuals with tetraplegia at model spinal cord injury systems. Spinal Cord 2004; 42: 169-176.

15 Afsar SI, Yemisci OU, Cosar SN, Cetin N. Compliance with clean intermittent catheterization in spinal cord injury patients: a long-term follow-up study. Spinal Cord 2013; 51: 645-649.

16 Seth JH, Haslam C, Panicker JN. Ensuring patient adherence to clean intermittent selfcatheterization. Patient Prefer Adher 2014; 8: 191-198.

$17 \mathrm{Kriz} \mathrm{J,} \mathrm{Relichova} \mathrm{Z.} \mathrm{Intermittent} \mathrm{self-catheterization} \mathrm{in} \mathrm{tetraplegic} \mathrm{patients:}$ a 6-year experience gained in the spinal cord unit in Prague. Spinal Cord 2014; 52 163-166. 